\title{
The Sitting-Unsupported Balance Score as an Early Predictor of Functional Prognosis in Stroke Patients: A Pilot Study
}

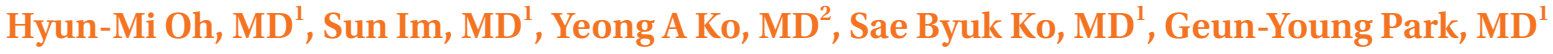

\begin{abstract}
${ }^{1}$ Department of Rehabilitation Medicine, Bucheon St. Mary's Hospital, The Catholic University of Korea College of Medicine, Bucheon; ${ }^{2}$ Baek Rehabilitation Hospital, Hwasun, Korea
\end{abstract}

\begin{abstract}
Objective To evaluate the impact of initial "sitting-unsupported" Berg Balance Scale (SUB), the specific trunk control parameter, on patients' functional outcome, Korean version of Modified Barthel Index (K-MBI) at 6 months.

Methods The charts of 30 patients retrospectively reviewed reviewed. The initial Korean version of Berg Balance Scale (K-BBS) including SUB along with patients' Korean version of Mini-Mental State Examination (K-MMSE), Glasgow Coma Scale (GCS), and other functional parameters that affect functional outcome were recorded. Cases were divided into low (group I) and high (group II) initial SUB score groups. Correlation and regression analysis were performed to assess the relationship between the initial SUB on the K-MBI at 6 months.

Results The mean \pm standard deviation score of initial SUB/K-MBI at 6 months of groups I and II were $0.056 \pm 0.236 / 26.89 \pm 32.48,3.58 \pm 0.515 / 80.25 \pm 18.78$, respectively, and showed statistical significant differences to each other $(\mathrm{p}<0.05)$. K-MBI at 6 months was highly correlated with initial GCS, SUB, K-BBS, K-MMSE, and initial K-MBI $(\mathrm{p}<0.05)$. In multiple linear regression analysis, initial SUB and GCS scores remained significantly associated with K-MBI at 6 months. A logistic regression model revealed that initial SUB ( $\mathrm{p}=0.004$, odds ratio=16), initial K-MBI, GCS, and K-MMSE were all significant predictors of K-MBI scores at 6 months.

Conclusion Initial SUB scores could be helpful in predicting patient's potential functional recovery at 6 months. Further studies with concurrent controls and a larger sample group are required to fully establish this tool.
\end{abstract}

Keywords Postural balance, Stroke, Prognosis, Rehabilitation

Received June 2, 2012; Accepted September 3, 2012

Corresponding author: Geun-Young Park

Department of Rehabilitation Medicine, Bucheon St. Mary's Hospital, The Catholic University of Korea College of Medicine, 327 Sosa-ro, Wonmi-gu, Bucheon 420-717, Korea

Tel: +82-32-340-2170, Fax: +82-32-340-2173, E-mail: rootpmr@catholic. ac.kr

(a) This is an open-access article distributed under the terms of the Creative Commons Attribution Non-Commercial License (http://creativecommons. org/licenses/by-nc/3.0) which permits unrestricted noncommercial use, distribution, and reproduction in any medium, provided the original work is properly cited.

Copyright $\odot 2013$ by Korean Academy of Rehabilitation Medicine

\section{INTRODUCTION}

Stroke is a leading cause of disability; therefore, it is important to develop individualized care plans for poststroke patients to minimize the impact of stroke for successful rehabilitation outcomes. Because a hemiplegic patient's quality of life depends on her or his ability to ambulate and to do activities of daily living (ADL), predicting functional recovery at the acute stage is very important. There has been extensive research on prediction 
of functional recovery [1]. Most research was based on multifactorial assessments, comparing the patients' state at admission and at discharge, age at onset, previous stroke history, urinary incontinence, mental status and orientation at onset, severity of paralysis at onset, ability to do ADL at onset, and sitting balance at onset as predictors for functional recovery $[2,3]$. However, half of stroke patients were able to ambulate within the first 3 months $[4,5]$, and improved significantly in most ADL within the first 6 months [5]. Therefore, we hypothesized that predicting the patients' functional outcomes in the first 6 months could be useful for a clinician to plan and treat stroke patients in their rehabilitation stages.

There are few reports predicting prognosis at subacute stage, but in 2003 Meijer et al. [6] found that urinary in-

Table 1. Sitting unsupported score in Berg Balance Scale

\begin{tabular}{cl}
\hline Score & \multicolumn{1}{c}{ Grade description } \\
\hline 4 & Able to sit safely and securely 2 minutes \\
3 & Able to sit 2 minutes under supervision \\
\hline 2 & Able to sit 30 seconds \\
\hline 1 & Able to sit 10 seconds \\
\hline 0 & Unable to sit without support 10 seconds \\
\hline
\end{tabular}

continence, initial ADL disability, ambulation, high age, severe paresis or paralysis, impaired swallowing, apraxia and stroke complication are possible predictors in the subacute stage in the systemic review. Sitting balance, one of the initial predictors, was positively correlated with final disability in stroke patients, and there was a positive correlation between sitting balance improvement and disabilities during the hospitalization [7]. There are, however, not many clinical tools to assess sitting balance of acute stroke patients. Therefore, the aim of the present study was to evaluate "sitting-unsupported" Berg Balance Scale (SUB), a subcomponent of the Korean version of Berg Balance Scale (K-BBS), as a predictor of functional outcome at subacute stage $[8,9]$ (Table 1).

\section{MATERIALS AND METHODS}

The medical records of thirty patients from October 2006 to February 2010 were reviewed. The protocol of this study was approved by the Institutional Review Board at the Catholic University of Korea, Bucheon St. Mary's Hospital, approval number HC11RISI0114. Inclusion criteria were first stroke attack, either cerebral infarct or cerebral hemorrhage, undergoing rehabilitation at our institution

Table 2. Demographic and clinical variables of participants

\begin{tabular}{lcccc}
\hline \multicolumn{1}{c}{ Characteristic } & Total $(\mathbf{n = 3 0})$ & Group I (n=18) & Group II (n=12) & p-value \\
\hline Age (yr) & $57.5 \pm 12.3$ & $57.6 \pm 13.8$ & $57.3 \pm 10.0$ & $0.285^{\text {a) }}$ \\
\hline Gender (male:female) & $19: 11$ & $12: 6$ & $7: 5$ & $0.752^{\text {b) }}$ \\
\hline Type of stroke (infarction:hemorrhage) & $7: 23$ & $3: 15$ & $4: 8$ & $0.405^{\text {b) }}$ \\
\hline K-MMSE & $8.833 \pm 10.89$ & $2.83 \pm 8.29$ & $17.83 \pm 7.697$ & $0.465^{\text {a) }}$ \\
\hline Dysphagia & 18 & 15 & 3 & $<0.0001^{\text {b) }}$ \\
\hline K-BBS & & & & \\
\hline Initial & $5.033 \pm 9.386$ & $0.056 \pm 0.236$ & $12.50 \pm 11.44$ & $<0.0001^{\text {a) }}$ \\
\hline After 6 mo & $25.37 \pm 21.61$ & $11.72 \pm 16.26$ & $45.83 \pm 7.791$ & $0.024^{\text {a) }}$ \\
\hline SUB & & & & \\
\hline Initial & $1.467 \pm 1.795$ & $0.056 \pm 0.236$ & $3.58 \pm 0.515$ & $<0.0001^{\text {a) }}$ \\
\hline After 6 mo & $2.700 \pm 1.760$ & $1.833 \pm 1.886$ & $4.00 \pm 0.001$ & $<0.0001^{\text {a) }}$ \\
\hline Initial GCS & $11.23 \pm 4.207$ & $9.278 \pm 4.254$ & $14.17 \pm 1.749$ & $0.001^{\text {a) }}$ \\
\hline K-MBI & & & & \\
\hline Initial & $16.23 \pm 20.44$ & $3.00 \pm 7.39$ & $36.08 \pm 17.34$ & $0.009^{\text {a) }}$ \\
\hline After 6 mo & $48.23 \pm 38.20$ & $26.89 \pm 32.48$ & $80.25 \pm 18.78$ & $0.006^{\text {a) }}$ \\
\hline
\end{tabular}

Values are presented as mean \pm standard deviation or number.

K-MMSE, Korean version of Mini-Mental Status Examination; K-BBS, Korean version of Berg Balance Scale; SUB, sitting-unsupported balance; K-MBI, Korean version of Modified Barthel Index; GSC, Glasgow Coma Scale.

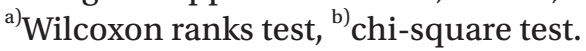


and having follow-up evaluation at 6 months post-stroke. Exclusion criteria were any orthopedic problem like fractures or any other combined neurologic diseases. Thirty patients (11 females and 19 males) were included in this study. Seven patients suffered from an ischemic accident, and 23 had a hemorrhagic stroke. The median age was 57.5 years, ranging from 34 to 89 years (Table 2).

The initial mental status of the patient was evaluated using Glasgow Coma Scale (GCS), and cognitive function was tested by Korean version of Mini-Mental State Examination (K-MMSE). Dysphagia was evaluated by videofluorographic swallowing study (VFSS). Functional ability was measured using the Korean version of Modified Barthel Index (K-MBI). In addition, the following were extracted from the patients' hospital records: initial KBBS including SUB and K-MBI at onset and at 6 months, K-MMSE, GCS at onset and other functional parameters known to affect functional outcome.

Cases were divided into low (score $\leq 2$; group I) and high (score $\geq 3$; group II) initial SUB score groups. Correlation and regression analysis were performed to assess the relationship between the initial SUB on the K-MBI at 6 months (Table 2).

The data were analyzed using the statistical package for the SAS ver. 9 (SAS Institute Inc., Cary, NC, USA). The differences between clinical functional parameters of groups I and II were evaluated using Wilcoxon signed rank test and chi-square test. To evaluate the distribution of initial functional parameters according to K-MBI after 6 months, the patients were divided into two groups according to K-MBI score at 6 months (cutoff value, 75). The difference was tested by using Wilcoxon ranks test and Fisher's exact test. The correlations between K-MBI and other functional parameters were tested using Spearman rank correlation coefficients and logistic regression. The Spearman rank correlation coefficient was selected because of the BBS's ordinal data scale.

\section{RESULTS}

This study included 30 stroke patients (group I, 18; group II, 12), divided into groups I and II by the initial SUB scores (group I, score $\leq 2$; group II, score $\geq 3$ ). The demographic and clinical variables of participants are summarized in the Table 2 . The differences were tested by using Wilcoxon ranks test and Fisher's exact test. Cor-
Table 3. Spearman correlation coefficients and p-value of variables to functional performance measure

\begin{tabular}{lcc}
\hline \multirow{2}{*}{ Variable } & \multicolumn{2}{c}{ K-MBI after 6 mo } \\
\cline { 2 - 3 } & Rho & p-value \\
\hline Age (time of stroke) & -0.238 & 0.238 \\
GCS & 0.691 & $<0.0001$ \\
K-MMSE & 0.727 & $<0.0001$ \\
Initial SUB & 0.746 & $<0.0001$ \\
Initial K-BBS & 0.748 & $<0.0001$ \\
Initial K-MBI & 0.619 & $<0.0001$ \\
\hline
\end{tabular}

Coefficients are shown between factors on initial state and scores of measures of functional performance at 6 months after stroke. K-MBI, Korean version of Modified Barthel Index at admission; GCS, Glasgow Coma Scale at initial physical examination; K-MMSE, Korean version of Mini-Mental State Examination for assessment of mental status; SUB, Sitting unsupported score in initial Berg Balance Score; initial K-BBS, total Korean version of Berg Balance Score at admission.

relation and regression analysis were performed to assess the relationship between the initial SUB on the K-MBI at 6 months (Table 2). The mean \pm standard deviation score of initial SUB/K-MBI at 6 months of groups I and II were $0.056 \pm 0.236 / 26.89 \pm 32.48,3.58 \pm 0.515 / 80.25 \pm 18.78$, respectively, and differed significant $(\mathrm{p}<0.05)$.

To show the relationship of variables to the functional performance measurements, Spearman correlation coefficients were evaluated. K-MBI at 6 months showed high correlations with initial GCS, SUB, K-BBS, K-MMSE, and initial K-MBI except age ( $\mathrm{p}<0.05)$ (Table 3). In multiple linear regression analysis, only initial SUB and GCS scores remained significantly associated with K-MBI at 6 months $\left(\mathrm{R}^{2}=0.69, \mathrm{p}<0.001\right)$. Other functional outcome measurements and K-MBI at 6 months did not show significant relationships in the multiple linear regression analysis.

To present the distribution of initial functional parameters according to K-MBI at 6 months, the patients were divided into two groups according to K-MBI score at 6 months (cutoff value, 75). Either Fisher's exact test or Wilcoxon ranks test was done on initial SUB, initial K-BBS, initial K-MBI, K-MMSE, dysphagia, and GCS. According to the distribution, there were significant relationships between K-MBI at 6 months and the initial functional parameters including initial SUB, K-BBS, K-MBI, K-MMSE, GCS and dysphagia (Table 4). 
Table 4. Distribution of initial functional parameters according to K-MBI after 6 months

\begin{tabular}{|c|c|c|c|c|}
\hline \multirow{2}{*}{ Parameter } & \multirow{2}{*}{ Total $(n=30)$} & \multicolumn{2}{|c|}{ K-MBI after 6 mo } & \multirow{2}{*}{ p-value } \\
\hline & & $<75(n=20)$ & $\geq 75(n=10)$ & \\
\hline SUB (initial) & & & & $0.004^{\mathrm{a})}$ \\
\hline$<3(0-2)$ & $18(60)$ & $16(80)$ & $2(20)$ & \\
\hline$\geq 3(3-4)$ & $12(40)$ & $4(20)$ & $8(80)$ & \\
\hline K-BBS (initial) & $0(0-33.0)$ & $0(0-30.0)$ & $7.0(0-33.0)$ & $0.0003^{\mathrm{b}}$ \\
\hline K-MBI (initial) & $2.5(0-58.0)$ & $0(0-58.0)$ & $33.0(7.0-54.0)$ & $0.001^{b)}$ \\
\hline K-MMSE & $0(0-28.0)$ & $0(0-24.0)$ & $20.0(0-28.0)$ & $0.002^{\mathrm{b})}$ \\
\hline Dysphagia & & & & $0.004^{\mathrm{a})}$ \\
\hline Yes & $18(60)$ & $16(80)$ & $2(20)$ & \\
\hline No & $12(40)$ & $4(20)$ & $8(80)$ & \\
\hline GCS & $13.0(3.0-15.0)$ & $10.0(3.0-15.0)$ & $15.0(9.0-15.0)$ & $0.022^{\mathrm{b})}$ \\
\hline
\end{tabular}

Values are presented as number (\%) or median (range).

SUB, sitting unsupported score in initial Berg Balance Score; K-BBS, total Korean version of Berg Balance Score at admission; K-MBI, Korean version of Modified Barthel Index at admission; K-MMSE, Korean version of Mini-Mental State Examination for assessment of mental status; GCS, Glasgow Coma Scale at initial physical examination.

${ }^{\text {a) }}$ Fisher's exact test, ${ }^{\text {b) }}$ Wilcoxon ranks test.

Table 5. Univariate logistic regression

\begin{tabular}{cccc}
\hline & \multicolumn{3}{c}{ Univariate logistic regression } \\
\cline { 2 - 4 } & OR & $\mathbf{9 5 \%}$ CI & p-value \\
\hline SUB & & $2.399-106.731$ & 0.004 \\
$<3(0-2)$ & 1.000 & & \\
$\geq 3(3-4)$ & 16.000 & & \\
K-BBS & & $1.790-201.681$ & 0.015 \\
$\quad<20$ & 1.000 & & \\
$\geq 20$ & 19.000 & & 0.012 \\
\hline K-MBI & & $1.637-53.205$ & \\
$<24$ & 1.000 & & 0.004 \\
\hline$\geq 24$ & 9.333 & & \\
\hline Dysphagia & & $2.399-106.731$ & \\
\hline Yes & 1.000 & & 0.015 \\
\hline No & 16.000 & & \\
\hline GCS & & $1.742-160.338$ & \\
\hline$<13$ & 1.000 & & \\
\hline 13 & 16.714 & & \\
\hline K-MMSE & & $1.125-31.989$ & 0.036 \\
\hline$<14$ & 1.000 & & \\
\hline$\geq 14$ & 6.000 & & \\
\hline
\end{tabular}

OR, odds ratio; CI, confidence interval; SUB, sitting unsupported score in initial Berg Balance Score; K-BBS, total Korean version of Berg Balance Score at admission; K-MBI, Korean version of Modified Barthel Index at admission; GCS, Glasgow Coma Scale at initial physical examination; K-MMSE, Korean version of Mini-Mental State Examination for assessment of mental status.
A univariate logistic regression analysis revealed that initial SUB ( $\mathrm{p}=0.004$, odds ratio $[\mathrm{OR}]=16$ ), initial K-BBS $(\mathrm{p}=0.015, \mathrm{OR}=19), \mathrm{K}-\mathrm{MBI}(\mathrm{p}=0.012, \mathrm{OR}=9.3)$, dysphagia $(\mathrm{p}=0.004, \mathrm{OR}=16), \mathrm{GCS}(\mathrm{p}=0.015, \mathrm{OR}=16.7)$, and K-MMSE $(\mathrm{p}=0.036, \mathrm{OR}=6)$, were all significant predictors of K-MBI scores at 6 months (Table 5). Group II (initial SUB $\geq 3$ ) had 16 times higher probability for K-MBI at 6 months to improve above 75 , compared with the Group I (initial SUB $<3$ ) (Table 5). In addition, a multivariate logistic regression analysis was done, and only initial MMSE was a significant predictor.

\section{DISCUSSION}

The aim of this present study was to evaluate the impact of the initial SUB, the specific trunk control parameter, on the patients' functional outcome as assessed by K-MBI at 6 months. We reviewed the medical records of all patients and recorded their initial K-BBS and K-MBI score at the time of admission and again at 6 months. As mentioned, initial SUB score was one of the significant predictors for functional improvement. It is important for a clinician to establish an individualized rehabilitation plan for a patient, predicting abilities to ambulate and to do ADL. Especially predicting the patients' functional outcomes at first 6 months is helpful for a clinician to set individualized goals in the treatment of stroke patients during rehabilitation. 
Trunk balance control is an essential motor ability for the execution of many functional tasks. The BBS was originally designed to evaluate balance in older adults [9]. The BBS involves minimal equipment and space and does not require special training. Although it allows quantitative assessment of balance for acute stroke patients, it is limited in that not all of the 14 items can apply to patients with complete loss of trunk balance or comatose patients. In this present study, we used early assessment of sitting balance (SUB) only to predict the functional outcome of stroke patients, and found a statistically significant relationship between SUB and K-MBI at 6 months.

As analyzing the data, there was a stronger relationship between initial SUB and K-MBI at 6 months than between K-BBS and K-MBI. This might be because K-MBI assesses ADL, which requires sitting balance for feeding and dressing. There are several studies that evaluated sitting balance as a predictor of the functional outcome [1012]. While several tools have been developed to assess sitting balance, there are no satisfactory tools available presently [13]. There are a number of scales including classic static, dynamic position balance, Trunk Impairment Scale [14], Clinical Outcome Variables Scale [15], Postural Assessment Scale for Stroke [16], and K-BBS. The BBS is a commonly used outcome tool in clinical practice [17], and the clinical usefulness of Korean version has also been proven [9]. Sitting unsupported position can be easily accessed by observing a patient sitting on a bed or a mat without support. It is scored from 0 to 4 , with a score of 0 representing the inability to sit without support and a score of 4 representing the ability to sit safely and securely for 2 minutes (Table 1). The SUB can easily be administered to all stroke patients with minimal space and time. Therefore, according to the results of this study, SUB can be helpful to predict the functional outcome of stroke patients.

There have been several published studies that stated $\mathrm{K}-\mathrm{BBS}$ is a reliable measurement for stroke patients with internal consistency. Jung et al. [8] stated that K-BBS was useful in predicting functional status at discharge in stroke patients, with a high relationship to Functional Independence Measure score. However, in this study, only K-MMSE has a statistically significant relationship with the K-MBI at 6 months on multivariate logistic regression analysis. This might be due to the characteristics of the study subjects, who had lower average K-BBS scores compared to those in other studies. In fact, patients with cognitive dysfunction were excluded in other studies [9]. Because our study included more severe stroke patients, as reflected by the low K-MMSE score, we believe that the SUB is more helpful in predicting the functional outcome of the patients than initial K-BBS for severe stroke patients as mentioned in the study of Juneja et al [18].

The limitations of the current study include possible selection bias due to its retrospective nature and the small number of study subjects. Therefore, we suggest a prospective randomized controlled test needs to be conducted. An additional limitation might be based on a small number of patients. As multivariate logistic regression was used to examine several possible predicting factors on K-MBI at 6 months, there was no statistically significant relationship between K-MBI at 6 months and SUB. However, Spearman correlation coefficients and the multiple linear regression analysis indicated that initial SUB remained statistically significant to K-MBI at 6 months. Therefore, it seems that the small number of patients included in this study influenced the results of the multivariate logistic regression test. Furthermore, a largescale prospective study should be conducted.

In this present study, we evaluated the impact of initial SUB, the specific trunk control parameter, on patients' functional outcome, K-MBI at 6 months. Besides, K-BBS, K-MBI, K-MMSE, dysphagia, and initial GCS score have a positive correlation with the functional outcome. Among several prognostic indicators, SUB can be used to evaluate balance abilities of acute stroke patients and as a predictor for subacute functional outcome with convenience. Further studies with concurrent controls and a larger sample group are recommended to fully establish this tool.

\section{CONFLICT OF INTEREST}

No potential conflict of interest relevant to this article was reported.

\section{ACKNOWLEDGMENTS}

Statistical consultation was supported by Catholic Research Coordinating Center of the Korea Health 21 R\&D Project (A070001), Ministry of Health \& Welfare, Republic 
of Korea.

\section{REFERENCES}

1. Jongbloed L. Prediction of function after stroke: a critical review. Stroke 1986;17:765-76.

2. Kim YC, Jang SJ, Park MY, Park SW. Prognostic factors of ambulation in stroke patients. J Korean Acad Rehabil Med 1992;16:443-51.

3. Kwakkel G, Wagenaar RC, Kollen BJ, Lankhorst GJ. Predicting disability in stroke: a critical review of the literature. Age Ageing 1996;25:479-89.

4. Skilbeck CE, Wade DT, Hewer RL, Wood VA. Recovery after stroke. J Neurol Neurosurg Psychiatry 1983;46:58.

5. Wade DT, Hewer RL. Functional abilities after stroke: measurement, natural history and prognosis. J Neurol Neurosurg Psychiatry 1987;50:177-82.

6. Meijer R, Ihnenfeldt DS, de Groot IJ, van Limbeek J, Vermeulen M, de Haan RJ. Prognostic factors for ambulation and activities of daily living in the subacute phase after stroke: a systematic review of the literature. Clin Rehabil 2003;17:119-29.

7. Duarte E, Marco E, Muniesa JM, Belmonte R, Diaz P, Tejero M, et al. Trunk control test as a functional predictor in stroke patients. J Rehabil Med 2002;34:26772 .

8. Jung HY, Kim TH, Park JH. Relationship between Berg balance scale and functional independence measure in stroke patients. J Korean Acad Rehabil Med 2005;29:167-70.

9. Lee JJ, Lee HJ, Park JH, Han EY, Kim MJ, Jung HY. The Korean version of Berg Balance Scale as an index of activity related to ambulation in subjects with stroke. J Korean Acad Rehabil Med 2007;31:400-3.
10. Sandin KJ, Smith BS. The measure of balance in sitting in stroke rehabilitation prognosis. Stroke 1990;21:826.

11. Feigin L, Sharon B, Czaczkes B, Rosin AJ. Sitting equilibrium 2 weeks after a stroke can predict the walking ability after 6 months. Gerontology 1996;42:348-53.

12. Verheyden G, Nieuwboer A, De Wit L, Feys H, Schuback B, Baert I, et al. Trunk performance after stroke: an eye catching predictor of functional outcome. J Neurol Neurosurg Psychiatry 2007;78:694-8.

13. Amusat N. Assessment of sitting balance of patients with stroke undergoing inpatient rehabilitation. Physiother Theory Pract 2009;25:138-44.

14. Verheyden G, Nieuwboer A, Mertin J, Preger R, Kiekens $C$, De Weerdt W. The trunk impairment scale: a new tool to measure motor impairment of the trunk after stroke. Clin Rehabil 2004;18:326-34.

15. Sacks L, Yee K, Huijbregts M, Miller PA, Aggett T, Salbach NM. Validation of the activity inventory of the Chedoke-McMaster stroke assessment and the clinical outcome variables scale to evaluate mobility in geriatric clients. J Rehabil Med 2010;42:90-2.

16. Benaim C, Perennou DA, Villy J, Rousseaux M, Pelissier JY. Validation of a standardized assessment of postural control in stroke patients: the Postural Assessment Scale for Stroke patients (PASS). Stroke 1999;30:1862-8.

17. Smith PS, Hembree JA, Thompson ME. Berg balance scale and functional reach: determining the best clinical tool for individuals post acute stroke. Clin Rehabil 2004;18:811-8.

18. Juneja G, Czyrny JJ, Linn RT. Admission balance and outcomes of patients admitted for acute inpatient rehabilitation. Am J Phys Med Rehabil 1998;77:388-93. 